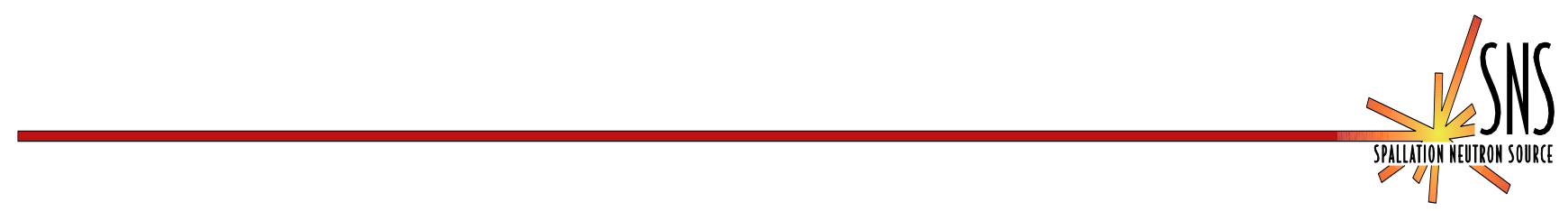

\title{
Labeling of Magnet Coil Leads \\ in accordance with \\ SNS Magnet Polarity Conventions
}

\author{
BNL/SNS Technical Note \\ No. 119
}

\author{
W. J. McGahern \\ November 14, 2002
}

Collider-Accelerator Department

Brookhaven National Laboratory

Upton, New York \#11973 


\title{
Labeling of Magnet Coil Leads \\ in accordance with \\ SNS Magnet Polarity Conventions
}

\author{
W. J. McGahern \\ November, 2002
}

\section{Introduction:}

References (1) and (2) define the convention that has been established for the determination of magnet polarities in the HEBT and Ring/RTBT beam lines.

In keeping with these established conventions, SNS main magnets have their coil leads labeled to provide clear identification to help ease confusion during electrical hook-ups in the field.

For SNS Ring and Transport System, all main magnets have their coil leads labeled as either $\# 1$ or $\# 2$. This labeling is used to indicate that the magnet will be in " $A$ " polarity, as viewed from the lead/connection end of the magnet, when terminal \#1 is connected to the positive (+) leg/terminal of the power supply.

This identification also holds true for the trim corrector windings when their polarity is viewed from the lead/connection end of the main (quadrupole) magnet.

\section{Field Layout (reprinted from (2)):}

The magnetic field lines for the multipoles are shown in the following schematics. The skew multipoles are obtained by rotating regular multipoles in the clockwise direction. The coordinate system is shown in Fig. 1, where $X$ is the radial direction, $Y$ is the vertical direction and $Z$ is along the beam. Fig. 2 shows the regular and skew dipoles, Fig. 3 shows the quadrupoles, Fig. 4 shows the sextupole fields and finally, Fig. 5 shows the octupoles. 


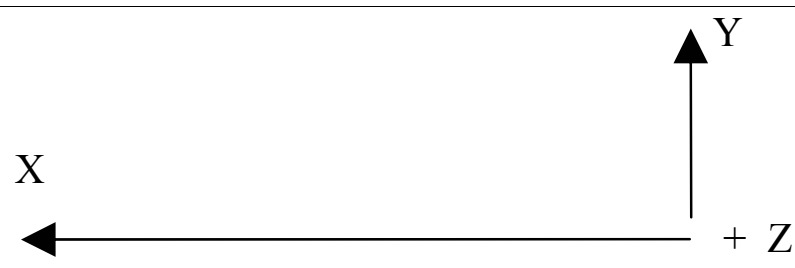

Figure 1: The coordinate system used. $X$ is radial, away from the center of the ring. $Z$ is into the paper in the clockwise direction around the accumulator ring.

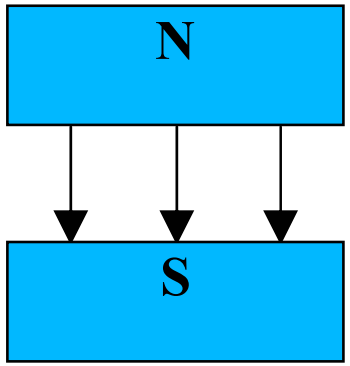

Regular

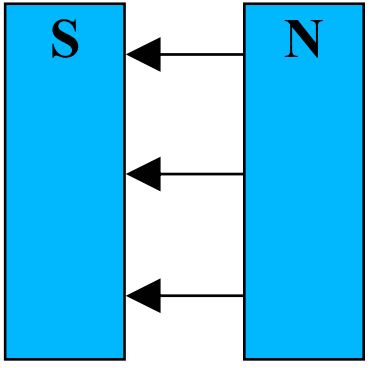

Skew

Figure 2: The field directions for the Horizontal (regular) and Vertical (skew) bending dipoles when the magnet is powered with polarity $\boldsymbol{A}$. Skew dipole: Clockwise rotation of $90^{\circ}$.
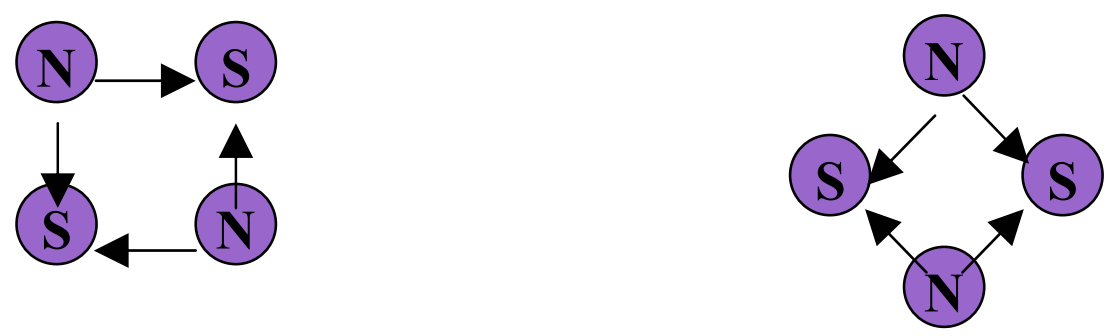

Regular

Skew

Figure 3: The regular and skew field lines for the quadrupoles in the accumulator ring. These field lines for polarity $A$. Skew quadrupole: Clockwise rotation of $45^{\circ}$. 


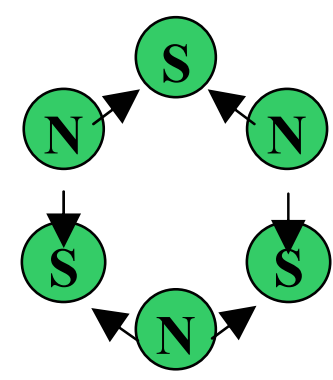

Regular

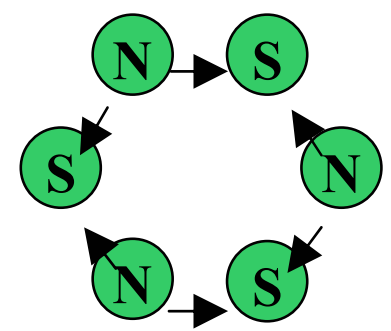

Skew

Figure 4: Sextupole field lines for regular and skew magnets. As above, these field lines are for polarity A. Skew sextupole: Clockwise rotation of $30^{\circ}$.

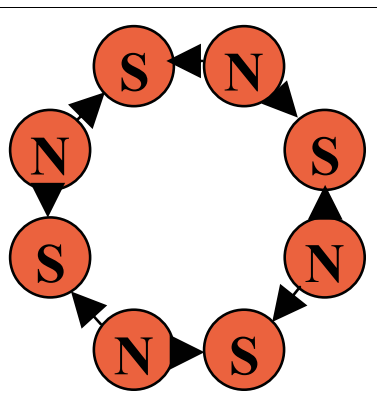

Regular

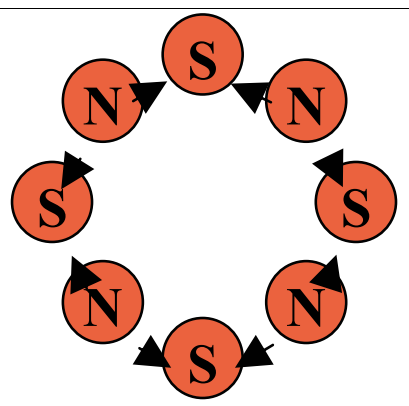

Skew

Figure 5: The regular and skew field lines for the octupole correction magnets. These are the field lines for polarity $\boldsymbol{A}$. Skew octupole: Clockwise rotation of $22.5^{\circ}$.

\section{Acknowledgments:}

A special thanks to Steve Tepikian, Y.Y. Lee, Deepak Raparia, Joe Tuozzolo and Bob Lambiase for all their help on the issues related to magnet/power supply hook-ups and polarities.

\section{Biography:}

(1) J. Wei, SNS Tech Note \#8, SNS Convention on Coordinate Systems, Console Display and Magnet Polarity, July 2001.

(2) S. Tepikian \& C. J. Gardner, SNS Tech Note \#114, Multipole Polarities for the SNS Accumulator Ring, July 2002. 